\title{
BMJ Open Key dimensions of post-traumatic stress disorder and endothelial dysfunction: a protocol for a mechanism-focused cohort study
}

\author{
Shiloh Cleveland, ${ }^{1}$ Kristina Reed, ${ }^{1}$ Jordan L Thomas, ${ }^{1}$ Olujimi A Ajijola, ${ }^{2}$ \\ Ramin Ebrahimi, ${ }^{3,4}$ Tzung Hsiai, ${ }^{3,5}$ Amit Lazarov, ${ }^{6}$ Amanda K Montoya, ${ }^{1}$ \\ Yuval Neria, ${ }^{7,8}$ Daichi Shimbo, ${ }^{9}$ Kate Wolitzky-Taylor, ${ }^{10}$ Jennifer A Sumner (D) ${ }^{1}$
}

To cite: Cleveland S, Reed K, Thomas JL, et al. Key dimensions of post-traumatic stress disorder and endothelial dysfunction: a protocol for a mechanism-focused cohort study. BMJ Open 2021;11:e043060. doi:10.1136/ bmjopen-2020-043060

- Prepublication history for this paper is available online. To view these files, please visit the journal online (http://dx.doi org/10.1136/bmjopen-2020043060).

Received 23 July 2020 Revised 24 February 2021 Accepted 16 April 2021

Check for updates

(C) Author(s) (or their employer(s)) 2021. Re-use permitted under CC BY-NC. No commercial re-use. See rights and permissions. Published by BMJ.

For numbered affiliations see end of article.

Correspondence to Dr Jennifer A Sumner; jsumner@psych.ucla.edu

\section{ABSTRACT}

Introduction Both trauma exposure and post-traumatic stress disorder (PTSD) are associated with increased risk of cardiovascular disease (CVD), the leading cause of death in the USA. Endothelial dysfunction, a modifiable, early marker of CVD risk, may represent a physiological mechanism underlying this association. This mechanismfocused cohort study aims to investigate the relationship between PTSD (both in terms of diagnosis and underlying symptom dimensions) and endothelial dysfunction in a diverse, community-based sample of adult men and women.

Methods and analysis Using a cohort design, 160 trauma-exposed participants without a history of CVD are designated to the PTSD group ( $\mathrm{n}=80$ ) or trauma-exposed matched control group $(n=80)$ after a baseline diagnostic interview assessment. Participants in the PTSD group have a current (past month) diagnosis of PTSD, whereas those in the control group have a history of trauma but no current or past psychiatric diagnoses. Endothelial dysfunction is assessed via flow-mediated vasodilation of the brachial artery and circulating levels of endothelial cell-derived microparticles. Two higher order symptom dimensions of PTSD — fear and dysphoria - are measured objectively with a fear conditioning paradigm and attention allocation task, respectively. Autonomic imbalance, inflammation, and oxidative stress are additionally assessed and will be examined as potential pathway variables linking PTSD and its dimensions with endothelial dysfunction. Participants are invited to return for a 2-year follow-up visit to reassess PTSD and its dimensions and endothelial dysfunction in order to investigate longitudinal associations.

Ethics and dissemination This study is conducted in compliance with the Helsinki Declaration and University of California, Los Angeles Institutional Review Board. The results of this study will be disseminated via articles in peer-reviewed journals and presentations at academic conferences and to community partners.

Trial registration number NCT03778307; pre-results.

\section{INTRODUCTION}

Despite advances in prevention and intervention, cardiovascular disease (CVD) remains
Strengths and limitations of this study

- This is the first study to assess endothelial dysfunction, an early marker of cardiovascular disease risk, using both functional (flow-mediated vasodilation) and cellular (endothelial cell-derived microparticle) approaches in a sample of trauma-exposed individuals.

- This study is the first to investigate whether posttraumatic stress disorder (PTSD) and its dimensions are related to change in endothelial dysfunction over time.

- This study comprehensively examines whether objective measures of underlying dimensions of PTSD - fear and dysphoria - are related to endothelial dysfunction.

- Whereas previous research on PTSD and endothelial dysfunction has been conducted in predominantly male samples of veterans and police officers, we have a diverse, community-based sample of men and women with and without current PTSD who have been exposed to a range of traumas.

- Although the approach of assessing key constructs in the research laboratory permits more controlled measures of these constructs, it limits the generalisability of findings to participants' experiences in real-world settings.

the leading cause of death in the USA. ${ }^{1}$ Growing research demonstrates longitudinal links between trauma exposure, posttraumatic stress disorder (PTSD) and CVD, suggesting trauma and PTSD as novel targets for reducing CVD risk. Trauma is common; the vast majority of individuals $(50 \%-90 \%)$ experience a trauma in their lifetime, including natural disasters, physical assault and unwanted sexual contact. ${ }^{23}$ PTSD is the quintessential trauma-related disorder, characterised by symptoms of re-experiencing of the trauma, avoidance of trauma reminders, negative alterations in cognition and mood, 
and hyperarousal. ${ }^{4}$ PTSD has been associated with heightened risk of incident CVD, even after accounting for numerous risk factors. ${ }^{5-11}$ Trauma exposure, independent of trauma-related psychopathology, has also been linked to elevated CVD risk. ${ }^{512-14}$

Given this evidence, experts in clinical psychology and cardiology have called for CVD risk surveillance after trauma. ${ }^{15}$ To support such efforts, it is critical to identify intermediary mechanisms linking post-traumatic stress and CVD risk that can guide monitoring and intervention approaches. Endothelial dysfunction may be one such mechanism. Endothelial cells, which form the inner lining of blood vessels, are one of the first physiological indicators of reduced capacity to respond to cardiovascular demand, with endothelial dysfunction implicated in the pathophysiology of atherosclerosis and CVD. ${ }^{16}{ }^{17}$ For every $1 \%$ decrease in brachial artery flow-mediated vasodilation (FMD; a functional measure of endothelial dysfunction), CVD risk increases by $9 \%-13 \% \cdot{ }^{18-20}$ Endothelial dysfunction can also be assessed by quantifying circulating levels of endothelial cell-derived microparticles (EMPs), direct measures of endothelial cell injury involved in atherosclerosis risk. ${ }^{21-24}$ As endothelial dysfunction can be detected in CVD-free individuals ${ }^{25}$ and is a malleable risk marker responsive to intervention, ${ }^{26}{ }^{27}$ it is an optimal target for prevention and risk mechanism research.

Initial studies in select samples (women aged 40-60 years, male veterans or police officers) suggest that trauma exposure and elevated PTSD symptoms are associated with lower FMD. ${ }^{28-30}$ Additionally, work in traumaexposed individuals reveals that the greater the PTSD symptom severity, the lower the FMD, even among individuals without PTSD diagnoses. ${ }^{28}$ PTSD has also been associated with biomarkers of endothelial dysfunction, ${ }^{31} 32$ although no studies have considered specific markers of cellular injury (EMPs).

Existing research has also been limited by treating PTSD as a homogeneous diagnosis. However, PTSD has great heterogeneity in how symptoms manifest. ${ }^{33}$ Thus, understanding which aspects of PTSD particularly affect intermediary mechanisms will inform monitoring efforts and intervention development. Broadly, PTSD consists of two higher order dimensions: fear and dysphoria. ${ }^{34-36}$ Fear reflects an alarm response to perceived danger, whereas dysphoria represents low positive affect and anhedonia. ${ }^{34}$ PTSD dimensions hold promise for distinguishing specific versus non-specific aspects of the disorder. ${ }^{34}$ Whereas dysphoria is considered an auxiliary component of PTSD, shared with depression, fear is considered a core component of PTSD. ${ }^{37}$ Longitudinal network analyses indicate that fear responses are central aspects of acute and chronic post-traumatic stress responses, whereas dysphoria symptoms are secondary responses that develop over time. ${ }^{35}$ Furthermore, impaired fear learning and inabilities to suppress fear in safe situations are key pathological processes in PTSD. ${ }^{38}$ Experimentally, fear conditioning paradigms provide a translational framework for examining dysregulated fear responses, ${ }^{39}$ which can be measured using objective psychophysiological measures, including skin conductance (SC) responses and fear-potentiated startle (FPS) ${ }^{40-42}$ In fear conditioning research, PTSD has been characterised by psychophysiological responses indicative of dysregulated fear responding, including difficulties with safety signal processing and fear inhibition. ${ }^{43-45}$ Additionally, some trauma-exposed individuals without PTSD exhibit psychophysiological responses akin to individuals with PTSD, ${ }^{46} 47$ reflecting similar trauma-related psychophysiological fear that could explain their lower FMD. ${ }^{28}$

Furthermore, psychophysiological fear responses are promising targets for understanding links between PTSD and CVD risk, as they may engage key biological mechanisms. For example, repeated bouts of psychophysiological reactivity may promote autonomic dysregulation $^{48-50}$ and elevated systemic inflammation and oxidative stress, ${ }^{51-53}$ which can have deleterious effects on cardiovascular health. ${ }^{54}$ However, research has yet to comprehensively examine mechanisms by which psychophysiological fear-or another PTSD dimension-may relate to endothelial dysfunction.

\section{Objectives}

This study examines links between PTSD and its dimensions with endothelial dysfunction in trauma-exposed men and women (see figure 1 for conceptual model). First, we examine the association of PTSD diagnosis with endothelial dysfunction, assessed via functional (FMD) and cellular (EMP) measures. We hypothesise that participants with versus without PTSD will have lower FMD and, secondarily, higher EMP levels. Second, we investigate which PTSD dimensions are most strongly associated with endothelial dysfunction. We predict that stronger psychophysiological fear responses (measured by FPS and SC) will be associated with lower FMD and, secondarily, higher EMP levels. Although we focus on the core dimension of fear, we investigate the dysphoria-endothelial dysfunction relation to comprehensively assess potential intervention targets. Third, we explore autonomic imbalance, inflammation and oxidative stress as potential mechanisms. Finally, we examine whether PTSD and its dimensions predict change in endothelial dysfunction over 2 years.

\section{METHODS AND ANALYSIS}

\section{Brief study overview}

This mechanism-focused cohort study examines primarily cross-sectional and, secondarily longitudinal, associations. Enrolment began in 2019, and the study is projected to continue through 2023. Investigating the PTSD diagnosis-endothelial dysfunction relation uses a case-control approach, whereas analyses of PTSD dimensions are conducted in the full sample. The sample will include 160 men and women without CVD from the Los Angeles area (80 individuals with PTSD; 80 traumaexposed controls). Group designations are determined 


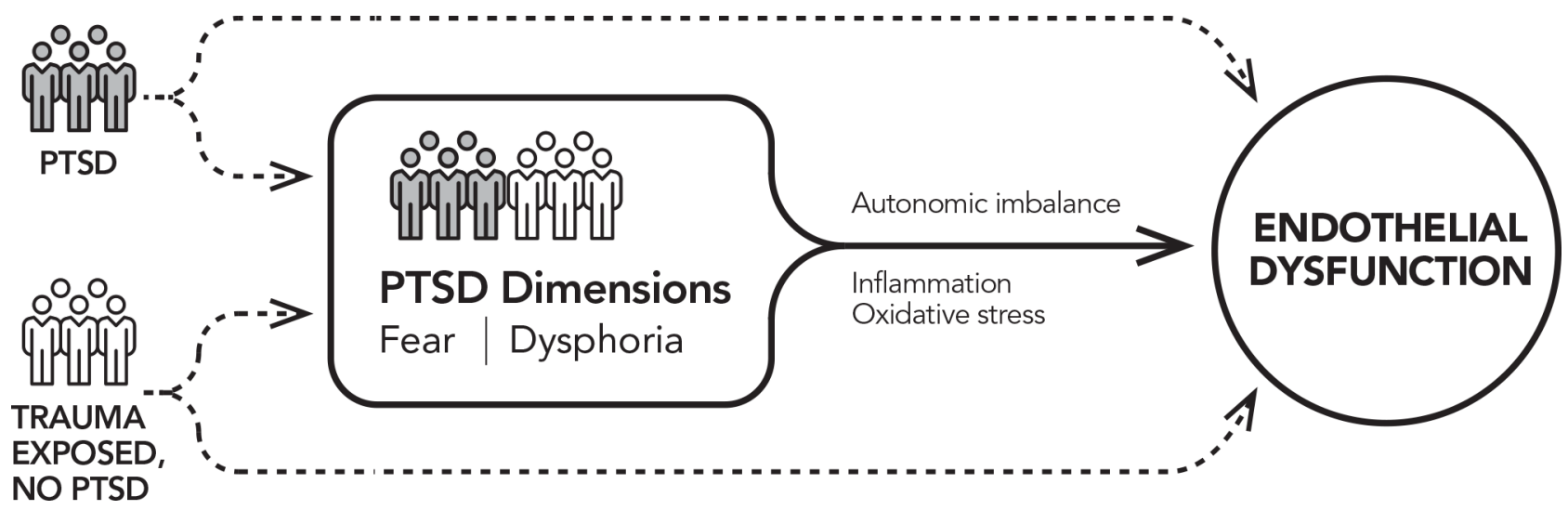

Figure 1 Conceptual model for the study. The aim examining whether PTSD diagnosis is related to endothelial dysfunction is indicated by the dashed arrows. The aim examining associations of post-traumatic fear and dysphoria with endothelial dysfunction is indicated by the solid arrow. Mechanisms examined in exploratory analyses are printed above and below the solid arrow. PTSD, post-traumatic stress disorder.

via diagnostic interview assessment. No-trauma controls were not included, as this study focuses on identifying what aspects of PTSD symptoms are linked to endothelial dysfunction.

\section{Participants}

Eligible participants are $\geq 18$ years old, fluent English speakers and trauma-exposed. Participants in the PTSD group have a current PTSD diagnosis based on the past-month Clinician-Administered PTSD Scale for Diagnostic and Statistical Manual of Mental Disorders, Fifth Edition (DSM-5) (CAPS-5), ${ }^{55}$ whereas controls are trauma exposed with no psychiatric diagnoses and a CAPS-5 score $<25 .{ }^{56}$ Exclusions include (a) individual CVD history (physician-diagnosed myocardial infarction, angina, heart failure, peripheral artery disease, stroke, transient ischaemic attack, atrial fibrillation, ventricular arrhythmias); (b) current psychotropic medication use (except benzodiazepines taken as needed), as this has been shown to influence key study variables including FMD, psychophysiological responses and attentional allocation $^{57-61}$; (c) current bipolar, psychotic, or moderate or severe substance use disorder; (d) mild or severe cognitive impairment (Mini-Mental State Examination (MMSE) score $\leq 18)^{62}$; (e) acute, unstable, or severe medical disorder or pregnancy; or (f) needing immediate psychiatric intervention (eg, active suicidality).

\section{Recruitment and enrolment}

The study timeline is depicted in figure 2. Participants are recruited via online advertisements, University of California, Los Angeles (UCLA) staff listservs, flyers throughout the community and word-of-mouth referrals. Interested individuals are phone screened to assess initial eligibility. After obtaining verbal consent, research personnel assess inclusion and exclusion criteria: English fluency, CVD history, psychotropic medication use, lifetime exposure to 17 types of trauma (using the Life Events Checklist for DSM-5), ${ }^{63}$ and past-month PTSD symptoms with respect to the trauma participants identify as most distressing (using the PTSD Checklist for DSM-5 (PCL-5)). ${ }^{64}$ The most distressing trauma is categorised as combat, non-combat interpersonal violence (eg, assault) or non-interpersonal violence (eg, accident). ${ }^{65}$ Time since trauma is also recorded. Eligible individuals are scheduled for a diagnostic interview assessment.

\section{Procedures}

Diagnostic interview assessment

Highly trained research personnel administer standardised clinical interviews. Interviewers have at least a Bachelor's degree and complete extensive training including self-guided study, didactics, diagnosing cases from recordings and role-playing. After obtaining written

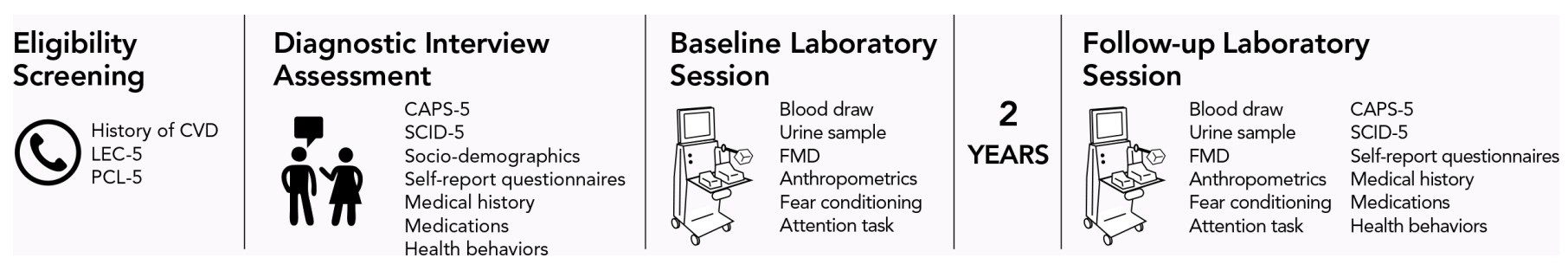

Figure 2 Study timeline. CAPS-5, Clinician-Administered PTSD Scale for DSM-5; CVD, cardiovascular disease; DSM-5, Diagnostic and Statistical Manual of Mental Disorders, Fifth Edition; FMD, flow-mediated dilation; LEC-5, Life Events Checklist for DSM-5; PCL-5, PTSD Checklist for DSM-5; PTSD, post-traumatic stress disorder; SCID-5, Structured Clinical Interview for DSM-5. 
informed consent, the interviewer conducts a brief clinical interview to build rapport and review information about the participant's history and current functioning.

The first diagnostic interview administered is the CAPS$5,{ }^{55}$ a gold standard structured interview querying pastmonth experiences of the 20 DSM-5 PTSD criteria. ${ }^{66}$ Symptoms are anchored to an index trauma self-identified by participants as their most distressing event. Responses for each symptom are scored with respect to frequency and intensity and combined into a severity score ranging from 0 (absent) to 4 (extreme/incapacitating). Symptom endorsement is used to determine current PTSD diagnosis, and severity scores are summed for a total severity score. Presence and history of other psychopathology are evaluated using the Structured Clinical Interview for DSM-5, Research Version, ${ }^{67}$ the gold standard in psychopathology assessment. Mood disorder episodes and psychotic symptoms are assessed for all participants; anxiety, substance abuse, eating and obsessive-compulsive disorders are assessed as needed based on screening questions. Interviewers then assess participants' medical history and health behaviours, including family history of CVD, personal history of medical conditions, pregnancy, medications, smoking and physical activity. If cognitive impairment is suspected, the MMSE is administered. ${ }^{62}$

Participants conclude the visit by completing several valid and reliable questionnaires. After reporting on sociodemographics, participants complete measures of childhood adversity: the Childhood Trauma Questionnaire ${ }^{68}$ (measuring child abuse and neglect); the Childhood Experiences of Care and Abuse ${ }^{69}$ antipathy and neglect items (anchored to participants' primary caregiver); the Conflict Tactics Scale ${ }^{70}$ (measuring family violence); and a food insecurity measure based on the US Department of Agriculture's Food Security Scale. ${ }^{71}$ Participants report on PTSD symptoms (anchored to the CAPS-5 index trauma) using the PCL-5 $5^{64} 72$ and depressive symptoms using the Patient Health Questionnaire-8. ${ }^{73}$ Participants describe their sleep with the Pittsburgh Sleep Quality Index (PSQI) ${ }^{74}$ PSQI Addendum for PTSD,${ }^{75}$ Insomnia Severity Index ${ }^{76}$ and RU-SATED scale. ${ }^{77}$

Propensity scores are used in recruitment to improve the balance of sociodemographic and trauma-related characteristics across the PTSD and trauma-exposed control groups. ${ }^{78}$ Scores reflecting propensity of PTSD group membership are calculated using age, gender, race/ethnicity, trauma type, time since trauma and twoway interactions among these variables. Matching during recruitment is based on quintiles of the propensity score distribution, with scores re-estimated weekly throughout recruitment. Participants are recruited if there are $<3$ unmatched individuals from their group in the same quintile.

Diagnostic interview assessments are reviewed by interviewers and supervised by a licensed clinical psychologist. Participants are informed of their eligibility via phone, with eligible participants scheduled for a laboratory visit $\sim 2-3$ weeks later.

\section{Baseline laboratory visit}

Participants are instructed to avoid exercise, food and drink (except water), smoking, and use of other tobacco, marijuana or cannabidiol-containing products at least 8 hours before this visit. Medications and vitamins (except diabetes medication, blood thinners, statins and birth control) are avoided for at least 48 hours.

Participants are asked to arrive at the research laboratory by $08: 00$. They are escorted to a study room for initial measurements and specimen collection; assessments are conducted by a research nurse. Height, weight, waist and hip circumferences, and left arm circumference are measured, and a spot urine sample is collected. An appropriately sized blood pressure cuff is placed on the left arm. After a 5-minute rest, three seated blood pressure measures are taken $1 \mathrm{~min}$ apart using a validated device (Omron HEM907XL). Blood is then drawn into serumseparating, citrate and EDTA tubes. The citrate tube is centrifuged immediately and processed to measure EMPs. EDTA tubes are centrifuged within 1 hour of collection at $1500 \mathrm{~g}$ at $4^{\circ} \mathrm{C}$ for $10 \mathrm{~min}$ to isolate plasma; aliquots are stored at $-80^{\circ} \mathrm{C}$ for inflammatory marker assays. Serum and whole blood are extracted from serum-separating and EDTA tubes for comprehensive metabolic and lipid panels and haemoglobin A1c, respectively.

Participants are then escorted to a temperaturecontrolled room to measure brachial artery FMD using a high-resolution, semiautomatic ultrasonography system (UNEXEF38G). ${ }^{79-81}$ Testing occurs between 08:00 and 10:00 to account for circadian effects on FMD and is conducted according to published guidelines. ${ }^{82} 83$ Participants lie supine, and a blood pressure cuff is placed around the right forearm. After a 15-minute rest, a baseline measure of brachial artery diameter is obtained. The UNEXEF38G system has a high-resolution linear artery transducer, coupled to computer-assisted analysis software that uses an automated edge detection system for measurement. The cuff is then inflated to $200 \mathrm{~mm} \mathrm{Hg}$ (or $50 \mathrm{~mm} \mathrm{Hg}$ above systolic pressure, if $>150 \mathrm{~mm} \mathrm{Hg}$ ) for $5 \mathrm{~min}$. Images of the brachial artery are obtained continuously until $2 \mathrm{~min}$ after cuff deflation.

After FMD measurement, participants are provided a snack before completing tasks assessing post-traumatic fear and dysphoria. Task order is counterbalanced. Prior to completing the fear conditioning paradigm used to assess post-traumatic fear, participants undergo a heart rate response to deep breathing (HRDB) protocol; both tasks involve psychophysiological measurements. HRDB tests autonomic function, specifically parasympathetic tone. ${ }^{84}$ Participants lie supine; respiration is measured with a respiration belt and ECG is measured with electrodes placed above the left and right collar bones and on the left lower forearm and recorded with the Biopac MP160 ECG wireless module. ECG and respiration data are collected during a 1-minute resting baseline and 10 -second respiratory cycle (5-second inhale, 5-second exhale), repeated six times. ${ }^{84}$ 
Post-traumatic fear is assessed using an established fear conditioning protocol with two phases: fear acquisition and extinction. ${ }^{45}$ During fear acquisition, participants complete a four-trial habituation phase in which two coloured shape conditioned stimuli (CS) and a $108 \mathrm{~dB}$, 40-millisecond broadband noise startle probe alone (NA) are presented to familiarise participants. Next, participants undergo a 36-trial conditioning phase, with 3 blocks of 12 trials: 4 reinforced CS (CS+), 4 non-reinforced CS (CS-) and 4 NA trials. CS are presented on a computer monitor for $6 \mathrm{~s}$; the startle probe is delivered binaurally via headphones on every trial after $6 \mathrm{~s}$. During conditioning, only the CS+ is reinforced with an unconditioned stimulus (US) on every trial. The US is a 250 -millisecond airblast of 140 psi intensity directed at the larynx, which has consistently produced robust FPS. ${ }^{43} 85$ On the CS+ trials, the US co-terminates with the CS+ $0.5 \mathrm{~s}$ following the startle probe; CS- trials terminate immediately after the startle probe. After a 10-minute break, participants undergo the 72-trial fear extinction phase: 6 blocks of 4 trials of each type (CS+, CS-, NA). During extinction, the CS+ is presented without the US. Intertrial intervals are randomised to be 9-22s for fear acquisition and extinction.

Psychophysiological fear responses are collected using Biopac MP160 electromyography (EMG) and SC wireless modules; ECG is also recorded. FPS is measured through corrugator and orbicularis EMG. Startle magnitude is assessed as the peak amplitude of EMG contraction $20-200 \mathrm{~ms}$ following the startle probe. SC data are collected using electrodes on the hypothenar surface of the non-dominant hand to measure sympathetic arousal. Psychophysiological data are filtered, rectified and smoothed using MindWare software. Several indicators of psychophysiological fear responses are calculated, including: (1) FPS to CS+ during early extinction (fear load), ${ }^{85}$ (2) FPS to CS+ duringlate extinction, and (3) FPS to CS- during late fear acquisition. These measures reflect expression of conditioned fear, fear inhibition and fear discrimination. ${ }^{85-87}$ FPS is calculated by subtracting startle magnitude to the NA from startle magnitude to the CS in each block to account for individual differences in startle magnitude and habituation. Comparable scores for SC response are secondary predictors. Although frequently used to index fear, SC response may be a more non-specific measure of arousal that is not as closely tied to fear neurocircuitry as FPS. ${ }^{87-89} \mathrm{SC}$ response is calculated by subtracting SC prior to stimulus onset from maximum SC during CS presentation.

Post-traumatic dysphoria is assessed with an established and reliable eye-tracking paradigm adapted for dysphoria. ${ }^{90}$ Attention allocation is measured using a remote high-speed eye-tracker (EyeLink Portable Duo). The task consists of free-viewing $4 \times 4$ matrices of NimStim ${ }^{91}$ happy and sad faces. Trials begin with a fixation cross, shown until a 1000-millisecond fixation is recorded, to verify trials begin with gaze fixated at the matrix's centre. Matrices are presented for 6000 milliseconds, followed by a 2000 -millisecond intertrial interval. Participants view 60 different matrices, presented in 2 blocks of 30 each, with a 60 -second break between blocks. Eye-tracking data are used to define fixations as $100+$ milliseconds of stable fixation within $1^{\circ}$ visual angle. Dwell time for two areas of interest (AOIs) - the eight sad faces and eight happy faces-is calculated for each matrix. Dwell time for each AOI is averaged across the 60 matrices.

\section{Follow-up visit}

Approximately 2 years after the baseline visit, all participants are invited to return for a follow-up; we aim to re-evaluate at least 80 individuals (40+ in each group). At follow-up, participants repeat all procedures from the baseline laboratory visit.

\section{Outcome measures}

Main outcomes

\section{Flow-mediated vasodilation}

Our primary outcome is brachial artery FMD. As described above, pulsed Doppler velocity signals are obtained at baseline and after cuff deflation using the UNEXEF38G ultrasonography system. FMD is the percent difference in brachial artery diameter, before and after occlusion.

\section{Endothelial cell-derived microparticles}

Circulating EMPs expressing CD62E and CD31 (endothelial cell activation and apoptosis, respectively ${ }^{24}$ assessed using flow cytometry are secondary outcome measures. EMP sample preparation is completed within 2 hours of blood draw, as previously described. ${ }^{92-95}$ Citrated blood is centrifuged at $160 \mathrm{~g}$ at $4^{\circ} \mathrm{C}$ for $10 \mathrm{~min}$ to obtain plateletrich plasma, which is further centrifuged at $1500 \mathrm{~g}$ at $4^{\circ} \mathrm{C}$ for $6 \mathrm{~min}$ to yield platelet-poor plasma. Fifty microlitres of platelet-poor plasma are each incubated in two sets: (1) $4 \mu \mathrm{L}$ of brilliant violet 421 (BV421)-conjugated monoclonal antibody to $\mathrm{CD} 62 \mathrm{E}$ (BD), $4 \mu \mathrm{L}$ of phycoerythrin (PE)-conjugated monoclonal antibody to CD31 (BD), $4 \mu \mathrm{L}$ of fluorescein isothiocyanate (FITC)-conjugated monoclonal antibody to $\mathrm{CD} 42 \mathrm{~b}(\mathrm{BD})$, and (2) $4 \mu \mathrm{L}$ of PE-conjugated monoclonal antibody to $\mathrm{CD} 31,4 \mu \mathrm{L}$ of FITC-conjugated annexin V (BD). EMPs are the number of particles with size $<1.5 \mu \mathrm{m}$ (1) positively labelled by CD62E, (2) positively labelled by CD31 and negatively labelled by CD42b, and (3) positively labelled by CD31 and annexin V. Allophycocyanin (APC)-conjugated monoclonal antibody CD45 (a pan-leucocyte marker; BD) is used to exclude contamination by leucocyte microparticles. ${ }^{93}$ Negative controls include the appropriate FITClabelled, PE-labelled, BV421-labelled and APC-labelled isotype-matched IgG. EMPs are quantified as the number of EMPs per microlitre (ThermoFisher Attune NxT Flow Cytometer).

\section{Potential pathway variables}

We are exploring autonomic imbalance, inflammation and oxidative stress as potential mechanisms linking PTSD and its dimensions with endothelial dysfunction. Average respiratory sinus arrhythmia amplitude across 
the cycles of the HRDB task-defined as the difference between the end of expiration and end of inspiration in heart rate-reflects parasympathetic-mediated cardiac control. ${ }^{84} 96$ Blood-based inflammatory markers are assayed from plasma, including high-sensitivity $\mathrm{C}$ reactive protein measured by ELISA (R\&D Systems). Interleukin (IL)-6, IL-8, IL-10, tumour necrosis factor- $\alpha$ and interferon-gamma are measured in a multiplex assay using a V-PLEX Custom Human Cytokine Proinflammatory Panel on the Meso Scale Discovery electrochemiluminesence platform. Oxidative stress is assessed by measuring urinary F2-isoprostanes using an ELISA (Oxford Biomedical Research).

\section{Statistical analyses}

We will first conduct cross-sectional associations of PTSD and its dimensions with endothelial dysfunction; longitudinal associations will be considered in exploratory analyses.

For cross-sectional analyses, two sample t-tests will compare participants with and without PTSD on (1) FMD and (2) EMPs expressing CD62E and CD31. We will extend these analyses using linear regression models adjusting for covariates (described below). We will consider total PTSD severity score as a continuous predictor. Second, objective measures of post-traumatic fear (eg, fear load based on FPS) will be the exposures in cross-sectional linear regression analyses of FMD and EMP outcomes. Additionally, we will explore objective measures of posttraumatic dysphoria as predictors of endothelial dysfunction. The approach for these analyses will mirror that for post-traumatic fear, with exposures operationalised as total dwell times for the sad and happy AOIs.

For longitudinal analyses, we will first examine how measures of PTSD and its dimensions at baseline predict change in FMD using linear regression, and then examine how changes in PTSD and its dimensions over follow-up predict change in FMD using linear regression. Analyses will consider EMPs as secondary outcomes. For longitudinal analyses, FMD or EMPs at follow-up will be the outcome; FMD or EMPs at baseline will be a covariate.

To examine if autonomic imbalance, inflammation and oxidative stress explain associations of PTSD and its dimensions with endothelial dysfunction in cross-sectional and/or longitudinal analyses, we will estimate total and direct effects of measures of PTSD and its dimensions on endothelial dysfunction and test for significant indirect effects using bootstrapping methods. ${ }^{97}$

For all analyses, we will examine a series of adjusted models. The base adjusted model will include age, gender, race and ethnicity; subsequent models will sequentially adjust for: (1) other sociodemographics (eg, education, marital status); (2) CVD medical risk factors (eg, diabetes, hypertension); (3) medications (eg, medication for hypertension, hyperlipidaemia); and (4) anthropometrics and health behaviours (eg, body mass index, smoking). Even though post-traumatic fear has been found to be distinct from the core symptoms of depression, ${ }^{42}{ }^{98}$ we will also consider depression as a potential confounder given high comorbidity of PTSD and depression. ${ }^{2}$ Data on depression diagnoses and symptoms will be examined as covariates, and we will explore how comorbidity of PTSD and other psychopathology (eg, depression) relates to endothelial dysfunction. To avoid overfitting in adjusted models, we will fit group lasso regression, which performs variable selection and regularisation of regression coefficients, to identify important predictors of endothelial dysfunction and improve model interpretability. ${ }^{99}$

\section{Sample size estimation and power}

Analyses are powered to our primary outcome, FMD, and based on the smallest effect size from prior research to be conservative in our calculations. We estimated our effect size based on Cohen's $d=0.48$ for the difference in per cent change in FMD in patients with versus without PTSD. ${ }^{28}$ Power analysis for two sample t-tests was conducted to determine a sufficient sample size using $\alpha=0.05$, power $=0.80$, a medium effect size (Cohen's $\mathrm{d}=0.48$ ), two tails, and equal allocations of participants to the PTSD and trauma control groups. Based on these assumptions, the desired sample size was 70 participants per group. We conservatively selected 80 participants per group.

Given our hypothesis that psychophysiological fear is the key PTSD dimension that will relate to endothelial dysfunction, we considered power for this analysis based on our primary exposure for this aim: FPS. We assumed an SD of 70 for FPS scores ${ }^{85}$ and an SD of $3.5 \%$ for FMD ${ }^{28}$ With $\mathrm{N}=160$ and a two-sided significance level of 0.05 , we have $80 \%$ power to detect a change of $0.011 \%$ in FMD for each unit of FPS score increase. ${ }^{100}$ This is a small effect size ${ }^{28}$ so our sample is large enough to model psychophysiological fear and FMD in adjusted models.

\section{Ethics and dissemination}

This study is conducted in compliance with the UCLA Institutional Review Board and Helsinki Declaration. Participants sign informed consent and Health Insurance Portability and Accountability Act Authorization for Research forms at enrolment. Research personnel review the study in detail and answer questions. Individuals are informed that participation is optional and that they may withdraw at any time. After individuals are fully informed about study procedures, participants who elect to proceed with the study sign the consent document, as do research personnel. The findings of this study will be disseminated online (ClinicalTrials.gov, Open Science Framework), presented at conferences and published in peer-reviewed journals.

\section{Patient and public involvement}

Patients and the public were not involved in the design or conduct of the study. We plan to share findings with the public through dissemination to community partners. 


\section{DISCUSSION}

This is the first study to comprehensively investigate the ways in which PTSD and dimensions of post-traumatic stress relate to endothelial dysfunction, one of the earliest modifiable precursors to CVD. To distil pathways by which post-traumatic stress may lead to CVD risk, we focus on psychophysiological fear-a key dimension of PTSD-although we consider objective measures of dysphoria, a more non-specific dimension. This study incorporates state-of-the-art measures of endothelial dysfunction by investigating brachial artery FMD and EMPs. No investigation of trauma-exposed individuals has considered functional and cellular measures of endothelial dysfunction as we do here. Furthermore, unlike prior research with predominantly white male samples, ${ }^{27}$ this study will include men and women of diverse racial/ethnic backgrounds who have been exposed to a range of traumas, increasing generalisability of findings.

Despite these strengths, there are limitations. Assessments are conducted in the laboratory, which offers a controlled environment that enhances internal validity but limits generalisability to real-world settings. Additionally, although we use state-of-the-art measures of endothelial dysfunction, neither metric indicates endothelial function reactivity to trauma-related cues. Further research using provocation measures or assessing cardiovascular reactivity in naturalistic settings is needed to address this issue.

PTSD predicts incident CVD, but the field needs intervention targets and intermediary mechanisms to determine if PTSD interventions can offset CVD risk. This study will test whether endothelial dysfunction could be an early subclinical, modifiable mechanism by which PTSD increases CVD risk, and whether post-traumatic fear or another dimension could be the target to offset that risk in vulnerable, trauma-exposed individuals in future intervention studies.

\section{Author affiliations \\ ${ }^{1}$ Psychology, University of California Los Angeles, Los Angeles, California, USA ${ }^{2}$ Neurocardiology Research Center of Excellence, Cardiac Arrhythmia Center, University of California Los Angeles, Los Angeles, California, USA \\ ${ }^{3}$ Medicine, University of California Los Angeles, Los Angeles, California, USA ${ }^{4}$ Medicine, Cardiology Section, Veterans Affairs Greater Los Angeles Healthcare System, Los Angleles, California, USA \\ ${ }^{5}$ Bioengineering, University of California Los Angeles, Los Angeles, California, USA ${ }^{6}$ School of Psychological Sciences, Tel Aviv University, Tel Aviv, Israel ${ }^{7}$ Psychiatry, Columbia University Irving Medical Center, New York, New York, USA ${ }^{8}$ Epidemiology, Columbia University Irving Medical Center, New York, New York, USA ${ }^{9}$ Medicine, Columbia University Irving Medical Center, New York, New York, USA ${ }^{10}$ Psychiatry and Biobehavioral Sciences, University of California Los Angeles, Los Angeles, California, USA}

Contributors JAS, DS, YN, AL, OAA, RE, TH and KW-T contributed significantly to the planning, conception, design and successful funding of this study. SC, KR, JLT, JAS and KW-T are contributing significantly to the acquisition of data. SC, KR and JAS drafted the initial version of this manuscript. AKM, DS, YN, AL, OAA, RE, TH and JAS will be involved in the analyses and interpretation of the data. All authors revised the draft critically for important intellectual content and gave final approval for this version of the manuscript to be submitted for publication.
Funding This study is supported by the National Heart, Lung, and Blood Institute (NHLBI) (grant number: R01HL139614).

Disclaimer The content is solely the responsibility of the authors and does not necessarily represent the official views of the National Institutes of Health.

Competing interests None declared.

Patient and public involvement Patients and/or the public were not involved in the design, or conduct, or reporting, or dissemination plans of this research.

Patient consent for publication Not required.

Provenance and peer review Not commissioned; externally peer reviewed.

Open access This is an open access article distributed in accordance with the Creative Commons Attribution Non Commercial (CC BY-NC 4.0) license, which permits others to distribute, remix, adapt, build upon this work non-commercially, and license their derivative works on different terms, provided the original work is properly cited, appropriate credit is given, any changes made indicated, and the use is non-commercial. See: http://creativecommons.org/licenses/by-nc/4.0/.

Author note $\mathrm{SC}$ and $\mathrm{KR}$ contributed equally to this work.

ORCID iD

Jennifer A Sumner http://orcid.org/0000-0002-0217-7171

\section{REFERENCES}

1 Virani SS, Alonso A, Benjamin EJ, et al. Heart disease and stroke Statistics - 2020 update: a report from the American heart association. Circulation 2020;141:e139-596.

2 Kessler RC, Sonnega A, Bromet E. Posttraumatic stress disorder in the National comorbidity survey. Arch Gen Psychiatry 1995;52:1048-60.

3 Kilpatrick DG, Resnick HS, Milanak ME, et al. National estimates of exposure to traumatic events and PTSD prevalence using DSM-IV and DSM-5 criteria. J Trauma Stress 2013;26:537-47.

4 American Psychiatric Association. Diagnostic and statistical manual of mental disorders, fifth edition (DSM-5). Washington, DC: American Psychiatric Association, 2013.

5 Sumner JA, Kubzansky LD, Elkind MSV, et al. Trauma exposure and posttraumatic stress disorder symptoms predict onset of cardiovascular events in women. Circulation 2015;132:251-9.

6 Kubzansky LD, Koenen KC, Jones C, et al. A prospective study of posttraumatic stress disorder symptoms and coronary heart disease in women. Health Psychol 2009;28:125-30.

7 Kubzansky LD, Koenen KC, Spiro A, et al. Prospective study of posttraumatic stress disorder symptoms and coronary heart disease in the Normative Aging Study. Arch Gen Psychiatry 2007:64:109-16.

8 Vaccarino V, Goldberg J, Rooks C, et al. Post-traumatic stress disorder and incidence of coronary heart disease. J Am Coll Cardiol 2013;62:970-8.

9 Boscarino JA. A prospective study of PTSD and early-age heart disease mortality among Vietnam veterans: implications for surveillance and prevention. Psychosom Med 2008;70:668-76.

10 Gradus JL, Farkas DK, Svensson E, et al. Associations between stress disorders and cardiovascular disease events in the Danish population. BMJ Open 2015;5:e009334.

11 Edmondson D, Kronish IM, Shaffer JA, et al. Posttraumatic stress disorder and risk for coronary heart disease: a meta-analytic review. Am Heart J 2013;166:806-14.

12 Scott KM, Koenen KC, Aguilar-Gaxiola S, et al. Associations between lifetime traumatic events and subsequent chronic physical conditions: a cross-national, cross-sectional study. PLoS One 2013;8:e80573.

13 Atwoli L, Platt JM, Basu A, et al. Associations between lifetime potentially traumatic events and chronic physical conditions in the South African stress and health survey: a cross-sectional study. BMC Psychiatry 2016;16:214.

14 Pietrzak RH, Goldstein RB, Southwick SM, et al. Physical health conditions associated with posttraumatic stress disorder in U.S older adults: results from wave 2 of the National Epidemiologic Survey on Alcohol and Related Conditions. J Am Geriatr Soc 2012;60:296-303.

15 Burg MM, Soufer R. Post-traumatic stress disorder and cardiovascular disease. Curr Cardiol Rep 2016;18:94.

16 Flammer AJ, Anderson T, Celermajer DS. The assessment of endothelial function: from research into clinical practice. Circulation 2012;126:753-67. 
17 Shimbo D, Grahame-Clarke C, Miyake Y, et al. The association between endothelial dysfunction and cardiovascular outcomes in a population-based multi-ethnic cohort. Atherosclerosis 2007:192:197-203.

18 Ras RT, Streppel MT, Draijer R, et al. Flow-mediated dilation and cardiovascular risk prediction: a systematic review with metaanalysis. Int J Cardiol 2013;168:344-51.

19 Matsuzawa Y, Kwon T-G, Lennon RJ, et al. Prognostic value of flow-mediated vasodilation in brachial artery and fingertip artery for cardiovascular events: a systematic review and meta-analysis. J Am Heart Assoc 2015;4:e002270.

20 Inaba Y, Chen JA, Bergmann SR. Prediction of future cardiovascular outcomes by flow-mediated vasodilatation of brachial artery: a meta-analysis. Int J Cardiovasc Imaging 2010;26:631-40.

21 Deanfield JE, Halcox JP, Rabelink TJ. Endothelial function and dysfunction: testing and clinical relevance. Circulation 2007;115:1285-95

22 Mallat Z, Benamer H, Hugel B, et al. Elevated levels of shed membrane microparticles with procoagulant potential in the peripheral circulating blood of patients with acute coronary syndromes. Circulation 2000;101:841-3.

23 Lynch SF, Ludlam CA. Plasma microparticles and vascular disorders. Br J Haematol 2007;137:36-48.

24 Jimenez JJ, Jy W, Mauro LM. Endothelial cells release phenotypically and quantitatively distinct microparticles in activation and apoptosis. Thromb Res 2003;109:175-80.

25 Celermajer DS, Sorensen KE, Georgakopoulos D, et al. Cigarette smoking is associated with dose-related and potentially reversible impairment of endothelium-dependent dilation in healthy young adults. Circulation 1993;88:2149-55.

26 Modena MG, Bonetti L, Coppi F, et al. Prognostic role of reversible endothelial dysfunction in hypertensive postmenopausal women. $J$ Am Coll Cardiol 2002;40:505-10.

27 Kitta Y, Obata J-ei, Nakamura T, et al. Persistent impairment of endothelial vasomotor function has a negative impact on outcome in patients with coronary artery disease. J Am Coll Cardiol 2009;53:323-30.

28 Grenon SM, Owens CD, Alley H, et al. Posttraumatic stress disorder is associated with worse endothelial function among veterans. J Am Heart Assoc 2016:5:e003010.

29 Violanti JM, Andrew ME, Burchfiel CM, et al. Posttraumatic stress symptoms and subclinical cardiovascular disease in police officers. Int J Stress Manag 2006;13:541-54.

30 Thurston RC, Barinas-Mitchell E, von Känel R, et al. Trauma exposure and endothelial function among midlife women. Menopause 2018;25:368-74.

31 Plantinga L, Bremner JD, Miller AH, et al. Association between posttraumatic stress disorder and inflammation: a twin study. Brain Behav Immun 2013;30:125-32.

32 von Känel R, Hepp U, Traber R, et al. Measures of endothelial dysfunction in plasma of patients with posttraumatic stress disorder. Psychiatry Res 2008;158:363-73.

33 Galatzer-Levy IR, Bryant RA. 636,120 ways to have posttraumatic stress disorder. Perspect Psychol Sci 2013;8:651-62.

34 Zoellner LA, Pruitt LD, Farach FJ, et al. Understanding heterogeneity in PTSD: fear, dysphoria, and distress. Depress Anxiety 2014;31:97-106.

35 Bryant RA, Creamer M, O'Donnell M, et al. Acute and chronic posttraumatic stress symptoms in the emergence of posttraumatic stress disorder: a network analysis. JAMA Psychiatry 2017;74:135-42.

36 Forbes D, Parslow R, Creamer M, et al. A longitudinal analysis of posttraumatic stress disorder symptoms and their relationship with fear and anxious-misery disorders: implications for DSM-V. J Affect Disord 2010;127:147-52.

37 Gros DF, Simms LJ, Acierno R. Specificity of posttraumatic stress disorder symptoms: an investigation of comorbidity between posttraumatic stress disorder symptoms and depression in treatment-seeking veterans. J Nerv Ment Dis 2010;198:885-90.

38 Foa EB, Kozak MJ. Emotional processing of fear: exposure to corrective information. Psychol Bull 1986:99:20-35.

39 Duits P, Cath DC, Lissek S, et al. Updated meta-analysis of classical fear conditioning in the anxiety disorders. Depress Anxiety 2015;32:239-53.

40 Jovanovic T, Kazama A, Bachevalier J, et al. Impaired safety signal learning may be a biomarker of PTSD. Neuropharmacology 2012:62:695-704

41 Jovanovic T, Norrholm SD. Neural mechanisms of impaired fear inhibition in posttraumatic stress disorder. Front Behav Neurosci 2011:5:44.
42 Jovanovic T, Norrholm SD, Blanding NQ, et al. Impaired fear inhibition is a biomarker of PTSD but not depression. Depress Anxiety 2010;27:244-51.

43 Norrholm SD, Jovanovic T, Olin IW, et al. Fear extinction in traumatized civilians with posttraumatic stress disorder: relation to symptom severity. Biol Psychiatry 2011;69:556-63.

44 Peri T, Ben-Shakhar G, Orr SP, et al. Psychophysiologic assessment of aversive conditioning in posttraumatic stress disorder. Biol Psychiatry 2000;47:512-9.

45 Blechert J, Michael T, Vriends N, et al. Fear conditioning in posttraumatic stress disorder: evidence for delayed extinction of autonomic, experiential, and behavioural responses. Behav Res Ther 2007;45:2019-33.

46 Roy MJ, Costanzo M, Leaman S. Psychophysiologic identification of subthreshold PTSD in combat veterans. Stud Health Technol Inform 2012;181:149-55.

47 Costanzo M, Jovanovic T, Norrholm SD, et al. Psychophysiological investigation of combat veterans with subthreshold post-traumatic stress disorder symptoms. Mil Med 2016;181:793-802.

48 Thayer JF, Yamamoto SS, Brosschot JF. The relationship of autonomic imbalance, heart rate variability and cardiovascular disease risk factors. Int J Cardiol 2010;141:122-31.

49 Ruiz-Padial E, Sollers JJ, Vila J, et al. The rhythm of the heart in the blink of an eye: Emotion-modulated startle magnitude covaries with heart rate variability. Psychophysiology 2003;40:306-13.

50 Melzig CA, Weike Al, Hamm AO, et al. Individual differences in fear-potentiated startle as a function of resting heart rate variability: implications for panic disorder. Int J Psychophysiol 2009;71:109-17.

51 O'Donovan A, Ahmadian AJ, Neylan TC, et al. Current posttraumatic stress disorder and exaggerated threat sensitivity associated with elevated inflammation in the mind your heart study. Brain Behav Immun 2017;60:198-205.

52 Michopoulos V, Rothbaum AO, Jovanovic T, et al. Association of CRP genetic variation and CRP level with elevated PTSD symptoms and physiological responses in a civilian population with high levels of trauma. AJP 2015;172:353-62.

53 Miller MW, Sadeh N, stress T. Traumatic stress, oxidative stress and post-traumatic stress disorder: neurodegeneration and the accelerated-aging hypothesis. Mol Psychiatry 2014;19:1156-62.

54 Wentworth BA, Stein MB, Redwine LS, et al. Post-traumatic stress disorder: a fast track to premature cardiovascular disease? Cardiol Rev 2013;21:16-22.

55 Weathers FW, Blake DD, Schnurr P. The clinician-administered PTSD scale for DSM-5 (CAPS-5). National Center for PTSD, 2013. Available: http://www.ptsd.va.gov

56 Schnurr PP, Chard KM, Ruzek JI, et al. Design of VA Cooperative Study \#591: CERV-PTSD, comparative effectiveness research in veterans with PTSD. Contemp Clin Trials 2015:41:75-84.

57 Hantsoo L, Czarkowski KA, Child J, et al. Selective serotonin reuptake inhibitors and endothelial function in women. Journal of Women's Health 2014:23:613-8

58 Grillon C, Chavis C, Covington MF, et al. Two-week treatment with the selective serotonin reuptake inhibitor citalopram reduces contextual anxiety but not cued fear in healthy volunteers: a fear-potentiated startle study. Neuropsychopharmacology 2009:34:964-71.

59 Ikawa M, Tabuse H, Ueno S, et al. Effects of combination psychotropic drug treatment on heart rate variability in psychiatric patients. Psychiatry Clin Neurosci 2001;55:341-5.

60 Wells TT, Clerkin EM, Ellis AJ, et al. Effect of antidepressant medication use on emotional information processing in major depression. AJP 2014;171:195-200.

61 Zhang L, Yu F, Hu Q, et al. Effects of SSRI antidepressants on attentional bias toward emotional scenes in first-episode depressive patients: evidence from an eye-tracking study. Psychiatry Investig 2020;17:871-9.

62 Rovner BW, Folstein MF. Mini-mental state exam in clinical practice. Hosp Pract 1987;22:99, 103, 06, 10.

63 Weathers F, Blake D, Schnurr P. The life events checklist for DSM-5 (LEC-5). National Center for PTSD, 2013. Available: http://www. ptsd.va.gov

64 Weathers F, Litz B, Keane T. The PTSD checklist for DSM-5 (PCL-5). National Center for PTSD, 2013. Available: http://www.ptsd.va.gov

65 Ozer EJ, Best SR, Lipsey TL, et al. Predictors of posttraumatic stress disorder and symptoms in adults: a meta-analysis. Psychol Bull 2003;129:52-73.

66 Weathers FW, Bovin MJ, Lee DJ, et al. The Clinician-Administered PTSD scale for DSM-5 (CAPS-5): development and initial psychometric evaluation in military veterans. Psychol Assess 2018:30:383-95. 
67 First M, Williams J, Karg R. Structured clinical interview for DSM5-Research version (SCID-5 for DSM-5, research version; SCID-5RV). Arlington, VA: American Psychiatric Association, 2015.

68 Bernstein DP, Fink L, Handelsman L, et al. Initial reliability and validity of a new retrospective measure of child abuse and neglect. AJP 1994;151:1132-6.

69 Bifulco A, Brown GW, Harris TO. Childhood experience of care and abuse (CECA): a retrospective interview measure. J Child Psychol \& Psychiat 1994;35:1419-35.

70 Straus MA. Measuring intrafamily conflict and violence: the conflict tactics (CT) scales. J Marriage Fam 1979;41:75-88.

71 Blumberg SJ, Bialostosky K, Hamilton WL, et al. The effectiveness of a short form of the household food security scale. Am J Public Health 1999;89:1231-4.

72 Bovin MJ, Marx BP, Weathers FW, et al. Psychometric properties of the PTSD Checklist for Diagnostic and Statistical Manual of Mental Disorders-Fifth edition (PCL-5) in veterans. Psychol Assess 2016;28:1379-91.

73 Kroenke K, Strine TW, Spitzer RL, et al. The PHQ-8 as a measure of current depression in the general population. $J$ Affect Disord 2009;114:163-73.

74 Buysse DJ, Reynolds CF, Monk TH, et al. The Pittsburgh sleep quality index: a new instrument for psychiatric practice and research. Psychiatry Res 1989;28:193-213.

75 Germain A, Hall M, Krakow B, et al. A brief sleep scale for posttraumatic stress disorder: Pittsburgh sleep quality index addendum for PTSD. J Anxiety Disord 2005;19:233-44.

76 Bastien C, Vallieres A, Morin CM. Validation of the insomnia severity index as an outcome measure for insomnia research. Sleep Med 2001;2:297-307.

77 Buysse DJ. Sleep health: can we define it? does it matter? Sleep 2014;37:9-17.

78 Johnson ES, Dickerson JF, Vollmer WM, et al. The feasibility of matching on a propensity score for acupuncture in a prospective cohort study of patients with chronic pain. BMC Med Res Methodol 2017;17:42

79 Takase B, Hattori H, Tanaka Y, et al. Acute effect of whole-body periodic acceleration on brachial flow-mediated vasodilatation assessed by a novel semi-automatic vessel chasing UNEXEF18G system. J Cardiovasc Ultrasound 2013;21:130-6.

80 Tomiyama $\mathrm{H}$, Kohro T, Higashi $\mathrm{Y}$, et al. A multicenter study design to assess the clinical usefulness of semi-automatic measurement of flow-mediated vasodilatation of the brachial artery. Int Heart $J$ 2012;53:170-5

81 Tomiyama H, Kohro T, Higashi Y, et al. Reliability of measurement of endothelial function across multiple institutions and establishment of reference values in Japanese. Atherosclerosis 2015;242:433-42.

82 Thijssen DHJ, Black MA, Pyke KE, et al. Assessment of flowmediated dilation in humans: a methodological and physiological guideline. Am J Physiol Heart Circ Physiol 2011;300:H2-12.

83 Corretti MC, Anderson TJ, Benjamin EJ. Guidelines for the ultrasound assessment of endothelial-dependent flow-mediated vasodilation of the brachial artery: a report of the International brachial artery reactivity Task force. J Am Coll Cardiol 2002;39:257-65.

84 Novak P. Quantitative autonomic testing. J Vis Exp 2011;53:e2502.

85 Norrholm SD, Glover EM, Stevens JS, et al. Fear load: the psychophysiological over-expression of fear as an intermediate phenotype associated with trauma reactions. International Journal of Psychophysiology 2015;98:270-5.

86 Jovanovic T, Ely T, Fani N, et al. Reduced neural activation during an inhibition task is associated with impaired fear inhibition in a traumatized civilian sample. Cortex 2013:49:1884-91.

87 Glover EM, Phifer JE, Crain DF, et al. Tools for translational neuroscience: PTSD is associated with heightened fear responses using acoustic startle but not skin conductance measures. Depress Anxiety 2011;28:1058-66.

88 Davis $\mathrm{M}$. The role of the amygdala in fear-potentiated startle: implications for animal models of anxiety. Trends Pharmacol Sci 1992;13:35-41.

89 Lonsdorf TB, Golkar A, Lindström KM, et al. BDNF val66met affects neural activation pattern during fear conditioning and $24 \mathrm{~h}$ delayed fear recall. Soc Cogn Affect Neurosci 2015;10:664-71.

90 Lazarov A, Ben-Zion Z, Shamai D, et al. Free viewing of sad and happy faces in depression: a potential target for attention bias modification. J Affect Disord 2018;238:94-100.

91 Tottenham N, Tanaka JW, Leon AC, et al. The NimStim set of facial expressions: judgments from untrained research participants. Psychiatry Res 2009;168:242-9.

92 Jy W, Horstman LL, Jimenez JJ, et al. Measuring circulating cellderived microparticles. J Thromb Haemost 2004;2:1842-3.

93 Bernal-Mizrachi L, Jy W, Jimenez JJ, et al. High levels of circulating endothelial microparticles in patients with acute coronary syndromes. Am Heart J 2003;145:962-70.

94 Garcia S, Chirinos J, Jimenez J, et al. Phenotypic assessment of endothelial microparticles in patients with heart failure and after heart transplantation: switch from cell activation to apoptosis. The Journal of Heart and Lung Transplantation 2005;24:2184-9.

95 Jimenez JJ, Jy W, Mauro LM, et al. Endothelial microparticles released in thrombotic thrombocytopenic purpura express von Willebrand factor and markers of endothelial activation. $\mathrm{Br} J$ Haematol 2003;123:896-902.

96 Porges SW. Orienting in a defensive world: mammalian modifications of our evolutionary heritage. A Polyvagal theory. Psychophysiology 1995;32:301-18.

97 Preacher KJ, Hayes AF. SPSS and SAS procedures for estimating indirect effects in simple mediation models. Behav Res Methods Instrum Comput 2004;36:717-31.

98 Baker DG, Nievergelt CM, O'Connor DT. Biomarkers of PTSD: neuropeptides and immune signaling. Neuropharmacology 2012;62:663-73.

99 Tibshirani R. Regression shrinkage and selection via the LASSO. $J$ of the Soc B 1996;58:267-88.

100 Neter J, Wasserman W, Kutner M. Applied linear regression models. Chicago, IL: Richard D. Irwin, Inc, 1983. 